\title{
Endocrine systems of the skin
}

\author{
Kamil Leis, Ewelina Mazur, Magdalena J. Jabłońska, Michalina Kolan, Przemysław Gałązka
}

Department of General and Oncological Paediatric Surgery, Antoni Jurasz University Hospital No. 1, Collegium Medicum in Bydgoszcz, Nicolaus Copernicus University in Toruń, Poland

Adv Dermatol Allergol 2019; XXXVI (5): 519-523

DOI: https://doi.org/10.5114/ada.2019.89502

\begin{abstract}
Apart from its protective function, the skin is also both hormonally and metabolically active. Many hormones are produced in the skin and the regulation of these changes is controlled, as are other organs, by the pituitary gland and hypothalamus. Under the influence of stress factors such as ultraviolet radiation, many reactions are stimulated. After exposure to light in keratinocytes apart from eumelanin, also proopiomelanocortin, dopamine, and opioid substances - $\beta$-endorphins are produced. The skin also has a hypothalamic-pituitary-adrenal axis homolog. A corticotropin-releasing hormone is produced and reacts with its skin receptors that lead to, among others, increasing vascular permeability or induce keratinocytes differentiation. A similar homologous system found in skin structures is the hypothalamic-pituitary-thyroid axis, which is expressed by the presence of e.g. thyroid-stimulating hormone (TSH) receptors or thyroglobulin genes. Our review presents selected hormonal skin systems, reactions within them and their products.
\end{abstract}

Key words: corticotropin-releasing hormone, proopiomelanocortin, $\beta$-endorphins, keratinocytes, glucocorticoids.

\section{Introduction}

The basic and best-known function of the skin is its role as a barrier between the internal and external environment. However, this organ remains hormonally and metabolically active. This results, among others, from constant changes in the environment and the stimuli to which the organ is exposed (e.g. solar radiation, mechanical injuries). It is also the site for transformation of many hormones, such as thyroxine or testosterone. These products are produced locally and secreted. They exert systemwide effects and are controlled by the same mechanisms as in the case of other organs, hypothalamus and pituitary gland. Local regulating systems are the adrenergic, cholinergic and serotoninergic systems. Afferent neural network fibers, found in the living layers of the skin, in addition to the central nervous system signal transmission, also take part in hormonal processes taking place in the skin [1-3].

\section{Influence of ultraviolet radiation on the production of $\beta$-endorphin and dopamine}

Ultraviolet (UV) radiation is $100-400 \mathrm{~nm}$ long and its main source is sunlight. The UV carcinogenic proper- ties in the case of basal cell carcinoma, squamous cell carcinoma and melanoma have been already confirmed [4-6]. Keratinocytes exposed to this radiation, through p-53 induction, stimulate the production of a compound called proopiomelanocortin, which in turn is transformed into a hormone responsible for the $\alpha$-melanocytes activation and results in the synthesis of eumelanin and hence tanning $[7,8]$.

This radiation also takes part in skin neuroendocrine processes. As a result of UV action, proopiomelanocortin is processed to various peptides, including opioid $\beta$-endorphins. This endogenous compound is present in the plasma at a level of 1-12 pM and has a high affinity for the $\mu$-receptor [7]. Based on studies conducted on mice, it was found that exposure to ultraviolet radiation results in an increase in $\beta$-endorphins in the body, while after discontinuation of rodent exposure, the level of this compound returned to its baseline level. The animals also showed an altered nociceptive threshold, quantified both by thermal and mechanical factors (analgesia). The use of naloxone prior to pain threshold testing resulted in results similar to the control, i.e. mice not exposed to UV irradiation [7]. This analysis also showed that UV ex-

Address for correspondence: Magdalena J. Jabłońska, Ludwik Rydygier Collegium Medicum, Nicolaus Copernicus University, 9 M. Curie Skłodowskiej St, 85-094 Bydgoszcz, Poland, phone: +48 5258540 15, e-mail: magdalena.jablonska14@gmail.com Received: 11.02.2019, accepted: 28.06.2019. 
posure leads to an increase in opioid receptor activity. It can, therefore, be concluded that the production of ultraviolet-induced $\beta$-endorphins underlies the relation with tanning $[7,8]$.

This compound, produced and secreted in melanocytes, located in the skin, has a stimulating effect on melanogenesis and, when found in hair follicles, in gp100 cells, may affect the autocrine growth of these structures. Beta-endorphin activated in keratinocytes by endothelin-1 or cannabinoids, stimulates G protein-coupled incipient-rectifying potassium channel (GIRKs) and B-type endothelin receptors ( $\left.E T_{B}\right)$ and thus mediates analgesia [3].

UV radiation type $B$ indirectly causes also an increase in the concentration of dopamine, according to Aubert et al., secreted mainly by the caudate nucleus. This is the result of increased $\beta$-endorphin levels. It leads to basal striatal post-synaptic dopamine D2/D3 receptors activation. It has also been proven that UV increases blood flow in this brain structure $[8,9]$.

\section{Skin hypothalamic-pituitary-adrenal axis homolog}

Physiologically the skin, due to the action of factors such as ultraviolet B radiation or stress, produces hormones of the hypothalamic-pituitary-peripheral gland axis and hypothalamic neurohormones, creating a homological system in relation to hypothalamic-pituitary-adrenal (HPA) [2, 10]. Like the hypothalamus, the skin (melanocytes, epidermis, vessels located in the skin) secrete $\mathrm{CRH}$ - corticoliberin (a corticotropin-releasing hormone). It is a substance, which is composed of 41 amino acids and regulates the body's response to stressful conditions and induces the production of such hormones as proopiomelanocortin and urocortin. The secretion of the former was recorded in keratocytes, melanocytes, fibroblasts and Langerhans cells. On the other hand, the secretion of the latter, which works in the skin via the corticoliberin receptor, has been observed, among others, in hair follicles, melanocytes, sweat glands, and epidermis [2, 3, $10,11]$. CRH binds to its CRH-R1 receptor, located in the corticotropic cell in the pituitary gland, which causes the conversion of proopiomelanocortin to corticotropin and its secretion into the blood. CRH also increases vascular endothelial permeability, has proinflammatory effects (although due to thermal damage it may act in the opposite way and inhibit inflammatory processes, e.g. by reducing vascular endothelial growth factor (VEGF) via the MAPK pathway and specific type 1 receptor, and also by modulating the activity of the nuclear factor $\kappa$-lightchain-enhancer of activated B cells (NF- $\mathrm{kB}$ ) protein complex and inhibition of interleukin $1 \beta$ ) and also induces the production of interleukins 6 and $11[2,11,12]$. In the skin it stimulates processes of keratinocytes differentiation by initiating the influx of calcium ions and creating a concentration gradient. Protein kinase C (PKC), phos- pholipase C, compounds of the activator protein-1 (AP-1) group, also take part in these processes, and changes in expression include compounds such as melanocortin 1 receptor (MC1R), urocortin receptors type 1, type 2 and type 3 or NR3C1 (glucocorticoid receptor). The CRH type 1 receptor, on the other hand, increases the concentration of inositol triphosphate and catalyses the binding of the compound from the AP-1 - Jun D family, which also affects the differentiation, shape, and granularity of keratinocytes. CRH also activates mast cells, causing them to degranulate, which has an inflammatory effect. Depending on the concentration, it blocks the cell cycle at the $\mathrm{GO} / 1$ phase as well as melanocyte apoptosis processes. It also influences the melanocytic synthesis of proopiomelanocortin (POMC) and its further transformation into melanocyte-stimulating hormone (MSH). In the hair structures, together with $\mathrm{POMC}$, it regulates the production of cortisol, which is synthesized from CRH [12-14]. This compound, acting on the mRNA level, also increases concentrations of $3 \beta$-hydroxysteroid dehydrogenase, which initiates steroidogenesis processes in sebaceous glands [15]. Until now, the presence of corticoliberin binding protein in skin layers has not been observed, therefore the researchers do not agree whether the compound is produced peripherally or centrally and then transported to the skin [12]. The dermal production of corticoliberin and proopiomelanocortin may be confirmed by the fact that in melanoma and basal cell carcinoma as well as in psoriasis-affected keratinocytes, an increased concentration of these compounds and their products is observed [16].

There are two types of $\mathrm{CRH}$ receptors found in the skin: CRH-R1 and CRH-R2. The first of these is divided into seven subtypes, marked by successive letters of the alphabet from "a" to " $h$ ". It occurs in most cells of all skin layers. The second one is divided into 3 subtypes: $\alpha, \beta$ and $\gamma$ and is present among others in sebaceous glands and hair follicles. The skin corticoliberin receptors belong to the G protein-coupled receptor (GPCR) group [3]. Their stimulation is correlated with the increase in CAMP and calcium concentrations [1]. A substitute for CRHR-1 is Urc-1, which is able to activate the response to a $\mathrm{CRH}$ dependent stress factor [13]. Expression of receptors is controlled by interleukin-1 via the NF- $\mathrm{BB}$ pathway (nuclear factor $\kappa$-light-chain-enhancer of activated B cells). Its effects have been confirmed in the paraventricular nucleus in the hypothalamus, myometrium, or the pituitary. In addition, tumor necrosis factor $\alpha$ (TNF- $\alpha$ ) and CAMP, corticosterone, lipopolysaccharide, interleukin-6 (IL-6), interleukin-4 (IL-4), and Urc-1 express the same regulative property. IL-6 stimulates mast cells to produce CRHR type 1 and type 2, whereas IL-4 and also lipopolysaccharide (LPS) only act on CRHR type 2 production without affecting CRHR type 1 concentrations. In addition to the above compounds, induction of $\mathrm{CRH}$ receptors in the pathogenesis of many skin diseases, for example, psoriasis, is mediated by substance P. It leads to an increase in CRHR 
type 1 concentrations and consequently TNF- $\alpha$, IL-8 and VEGF with the participation of the neurokinin type 1 receptor [13].

Due to the action of stressors, a system called skin stress response system (SSRS) induces an increase in $\mathrm{CRH}$ levels, and thus proopiomelanocortin, which in turn is cleaved, with the participation of 7B2 protein and the enzymes proprotein convertase 1 and proprotein convertase 2 , to melanotropin, corticotropin, $\beta$-lipotropin, and $\beta$-endorphin $[13,16]$.

One of the stress factors inducing $\mathrm{CRH}$ skin production is UV. Zbytek et al. in 2006 published the results of the in vitro study on the effects of ultraviolet $B$ radiation on $\mathrm{CRH}$ POMC. In melanocytes ultraviolet led to induction of the corticoliberin promoter (mediated by the type A protein kinase pathway), POMC promoter (mediated by CRH-R1 receptor) and mRNA (both $\mathrm{CRH}$ and POMC), CAMP response element - binding protein phosphorylation (CREB) and its binding to CAMP response element (CRE) as well as secretion of tyrosinase-dependent proteins and ACTH [10]. In a study conducted by Skobiowat and Slominski on a mouse model conducted in 2015, it was found that type $\mathrm{B} U \mathrm{~V}$ radiation activates the HPA axis. After $12 \mathrm{~h}$ and $24 \mathrm{~h}$ of irradiation at a dose of $400 \mathrm{~mJ} / \mathrm{cm}^{2}$ and $290-320 \mathrm{~nm}$ wavelength, plasma, brain, adrenal, and skin samples were taken to measure concentrations of $\beta$-endorphin, corticoliberin, corticosterone, corticotropin, and urocortin. An increase in plasma levels of such hormones was observed: urocortin, $\beta$-endorphins, corticosterone, corticoliberin and corticotropin. The adrenal glands, subjected to UV type B irradiation, were characterized by increased mRNA concentration, which belonged to steroidogenic acute protein regulators, melanocortin receptor 2 and the gene responsible for steroid $11 \beta$-hydroxylase coding. The skin has increased concentrations of $\beta$-endorphins, corticosterone, and urocortin. It was also found that in the paraventricular nucleus, located in the hypothalamus, the $\mathrm{CRH}$ gene was expressed [11].

Kimura et al. in 2012 analysed the response of this system to trichloroacetic acid (TCA), which works by causing epidermal necrosis and its subsequent reconstitution including the matrix (a possible mechanism is the induction of interleukin 1, 10 and platelet growth factor B (PDGF-B)). These researchers showed that this agent, widely used in cosmetic procedures, activates skin POMC in a $\mathrm{CRH}$-independent manner. For this purpose, they took and compared the murine and human skin tissue samples, previously treated with TCA. Enhanced POMC and RNA proteins belonging to Mcr1 (mobilized colistin resistance) and $\mathrm{CRH}$ (in samples derived from human skin) and no increase in the concentration of proopiomelanocortin products as well as CRH and its skin receptors was observed [16].

In 2014, Pang et al. studied the reactions of the skin HPA system in response to the stress factor, which was corticosterone. Mice from the C57BL/ 6 line were divided into two groups: the control and the drug receivers. In the rodent test group, a decrease in melanin concentrations was observed, i.e. weakening of melanogenesis processes and suppression of reaction in the skin HPA system. Stress conditions also influenced the decrease in the expression of type 2 melanocortin receptors (which is, together with the melanocortin type 1 receptor, through which alphamelanotropin acts, a part of a transmembrane rhodopsinlike receptor group), to which ACTH and CYP11A1 P450scc (cytochrome P450 11A1) bind, the enzyme responsible for corticosteroids formation. These changes, however, were reversed after the use of the glucocorticoid receptor antagonist RU486, mifepristone, which blocked the corticosterone. Exposure to chronic stress factors, therefore, causes inhibition rather than induction of the HPA system as shown in previously described studies. Researchers have found that this may be due to the glucocorticoiddependent negative feedback [12, 17].

The dermal HPA system is also involved in the pathogenesis of many skin diseases associated with exposure of the body to stress. These include, among others, atopic dermatitis, psoriasis, alopecia areata, seborrhoea or skin cancer. It is associated with high levels of glucocorticoids, $\mathrm{CRH}$, vasopressin and many processes specific for a given disease entity. ACTH via the melanocortin receptor, p38, MAPK/ERK, and caspase-1 pathways stimulates cells from the HaCaT-immortal keratinocyte line to produce interleukin 18, which also participates in inflammatory processes. On the other hand, corticotropin has opposite effects, reducing its concentration with the p38 mitogenactivated protein kinases pathway $[12,18]$.

\section{Glucocorticosteroids formation in the skin}

In the skin, as in the adrenal cortex, there are processes of corticosteroids formation, which have both local and system-wide effects. They are characterized by paracrine, autocrine and intracrine effects, although endocrine activity has not been proven. The CYP11A1 isoenzyme works on cholesterol, initiating the synthesis of these substances. The pregnenolone neuro-steroid is converted by means of 3-hydroxysteroid dehydrogenase to 18-hydroxydeoxycorticosterone and deoxycorticosterone and then to corticosterone. Cortisol is produced by the conversion of 11-deoxycortisol or cortisone by the CYP11B1 and 11-HSD1 isoenzymes (11 $\beta$-hydroxysteroid dehydrogenase type 1), respectively. In addition to the abovementioned, CYP17A1, CYP21A2 and CYP11A11 isoenzymes as well as Star (steroidogenic acute regulatory protein), STARD3 (StAR-related lipid transfer domain protein 3), also under another name of MLN64 (metastatic lymph node 64 protein), are involved in the metabolism as well $[13,19]$.

The skin is also the site of the synthesis of steroid sex hormones that are produced from compounds such as androstenedione, dehydroepiandrosterone and dehy- 
droepiandrosterone sulfate. Dehydroepiandrosterone is metabolized to androstenediol and 4-androstenedione and finally to $5 \alpha$-dihydrotestosterone [13]. It has also been proven that human keratinocytes convert reversibly 17-estradiol to oestrone, whereas fibroblasts and adipocytes have the ability to produce estradiol. CYP11A1 can generate compounds belonging to the group of 7-steroids, which can be metabolized under the influence of the stress factor, e.g. ultraviolet radiation, to the analogues of vitamin D [13].

The intensity and efficiency of these processes occurring in the skin, as in the brain or heart, are at a low level. It has been proven that fibroblasts, melanocytes and also keratinocytes, found in the layers of the epidermis and in the hair follicles have the ability to produce glucocorticoids. The use of steroid sulfatase, however, enables the control of these processes. The activity of steroid products depends on GR receptors, which can be divided into $\alpha$ and $\beta$ and their action may involve, among others, MAPK or PI3K routes [19].

After skin exposure to ultraviolet radiation B, 7-dehydrocholesterol is converted to lumisterol, tachysterol or to vitamin $\mathrm{D}_{3}$. In keratinocytes 7-dehydrocholesterol, with the participation of CYP11A1, can be transformed to 22-hydroxy-7-dehydrocholesterol, 20,22-hydroxy-7dehydrocholesterol and finally to 7-dehydropregnenolone. The last one can be further metabolized until 7-steroid compounds and secosteroids are produced. The action of CYP11A1 and CYP27A1 is also possible by the formation of 25-hydroxy-7-dehydrocholesterol, 26-hydroxy7-dehydrocholesterol, 27-hydroxy-7-dehydrocholesterol, 25-hydroxycholesterol, 26-hydroxycholesterol, and 27-hydroxycholesterol [19]. Cutaneous steroid metabolism is also associated with the pathogenesis of many skin diseases. For example, high concentrations of 11b-hydroxysteroid dehydrogenase are observed in the sebaceous glands in the case of acne. In SZ95 sebocytes blocking this enzyme gene expression by small interfering RNA (siRNA) resulted in the inhibition of sterol regulatory element-binding protein-1, which subsequently reduced the intensity of lipogenesis processes in the course of psoriasis [15]. Also in the dermal tissues covered with hair, found on the head of men suffering from androgenic alopecia, an elevation of 11b-hydroxysteroid type 1 dehydrogenase and StAR was observed. The activity of the latter is also disturbed in eczema or seborrheic keratosis. In the case of basal cell carcinoma and squamous cell carcinoma of the skin, however, a weakened expression of 11b-hydroxysteroid dehydrogenase is observed $[15,19]$. In the course of psoriasis, keratinocytes are characterized by a low concentration of CCHCR1 (coiledcoil $\alpha$-helical rod protein 1 ). During wound healing, the expression of the above enzyme increases [15]. Slominski and Zmijewski in their work of 2017, citing studies by Canica et al., claim that glucocorticosteroids negatively affect wound regeneration by inducing the nongenomic glucocorticoid signal transduction. These researchers have demonstrated that dexamethasone, by binding its membrane receptors, stimulates phospholipase C and protein kinase $C$ signalling system. This results in the inhibition of glycogen synthase kinase $3 \beta$, leading to the activation of c-myc as well as $\beta$-catenin and the disruption of keratinocyte migration. The consequence of the above-mentioned processes is the healing of the wound [20].

\section{Hypothalamic-pituitary-thyroid axis skin homolog}

The skin is commonly affected in the thyroid diseases. Slominski et al. in 2002 observed that thyroid stimulating mRNA receptors - TSHR, sodium iodide symporter and deiodinase 2 and deiodinase 3 were present in keratinocytes, fibroblasts, melanocytes, hair follicle cells, and epidermis. Thyroglobulin and TSH- $\beta$ genes were also found. This may be a result of the homolog hypothalamic-pituitary-thyroid axis function. The TSH receptor detected by the researchers has shown to be functionally active, which could explain the pathogenesis of skin lesions in the course of Graves' disease [21].

Similar results were provided by the 2005 study by Agretti et al. They found that TSH receptor mRNA was present in the fibroblasts of people with the current manifestation of ophthalmopathy [22].

\section{Summary}

The skin is an organ that not only has a protective function, but is also the place of many metabolic changes and processes that are often influenced by external factors. One of them is ultraviolet radiation type $B$, which affects not only sunbathing, but also the production of POMC, dopamine, and beta-endorphins, which are a source of relation with tanning. Another system functioning in the dermal structures is the HPA - hypothalamic-pituitary-adrenal homolog axis. The skin produces corticotropin, which has a number of functions, e.g. it affects the vascular endothelium. In addition to the axis mentioned above, the skin is also the site of the homological hypothalamic-pituitary-thyroid axis. Therefore, the skin is not only a barrier separating the external and internal environment, but also the place of many important reactions and pathways, which are often involved in the pathogenesis of skin and allergic diseases. Many mechanisms are still unknown and further studies of many systems and hormonal processes taking place in the skin are necessary.

\section{Conflict of interest}

The authors declare no conflict of interest. 


\section{References}

1. Slominski A, Wortsman J. Neuroendocrinology of the skin. Endocr Rev 2000; 21: 457-87.

2. Swider-Al-Amawi M, Marchlewicz M, Kolasa A, et al. Neuroendokrynna funkcja skóry. Post Biol Komórki 2010; 4: 795-806.

3. Roosterman D, Goerge T, Schneider SW, et al. Neuronal control of skin function: the skin as a neuroimmunoendocrine organ. Physiol Rev 2006; 86: 1309-79.

4. Moan J, Grigalavicius M, Baturaite Z, et al. The relationship between UV exposure and incidence of skin cancer. Photodermatol Photoimmunol Photomed 2015; 31: 26-35.

5. Natarajan VT, Ganju P, Ramkumar A, et al. Multifaceted pathways protect human skin from UV radiation. Nat Chem Biol 2014; 10: 542-51.

6. Available at: http://www.spacewx.com/pdf/SET_ 21348_2004.pdf. Accessed: 30.01.2019.

7. Fell GL, Robinson KC, Mao J, et al. Skin beta-endorphin mediates addiction to UV light. Cell 2014; 157: 1527-34.

8. Tejeda HA, Bonci A. Shedding "UV" light on endogenous opioid dependence. Cell 2014; 157: 1500-1.

9. Aubert PM, Seibyl JP, Price JL, et al. Dopamine efflux in response to ultraviolet radiation in addicted sunbed users. Psychiatry Res Neuroimaging 2016; 251: 7-14.

10. Zbytek B, Wortsman J, Slominski A. Characterization of a ultraviolet B-induced corticotropin-releasing hormone-proopiomelanocortin system in human melanocytes. Mol Endocrinol 2006; 20: 2539-47.

11. Skobowiat C, Slominski AT. UVB activates hypothalamic-pituitary-adrenal axis in C57BL/6 mice. J Invest Dermatol 2015; 135: 1638-48.

12. Kim JE, Cho BK, Cho DH, Park HJ. Expression of hypothalamic-pituitary-adrenal axis in common skin diseases: evidence of its association with stress-related disease activity. Acta Derm Venereol 2013; 93: 387-96.

13. Slominski AT, Zmijewski MA, Zbytek B, et al. Key role of CRF in the skin stress response system. Endocr Rev 2013; 34: 827-84.

14. Wierzbicka JM, Żmijewski MA, Antoniewicz J, et al. Differentiation of keratinocytes modulates skin HPA analog. I Cell Physiol 2017; 232: 154-66.

15. Nikolakis G, Zouboulis CC. Skin and glucocorticoids: effects of local skin glucocorticoid impairment on skin homeostasis. Exp Dermatol 2014; 23: 807-8.

16. Kimura A, Kanazawa N, Li HJ, et al. Influence of chemical peeling on the skin stress response system. Exp Dermatol 2012; 21 Suppl 1: 8-10.

17. Pang S, Wu H, Wang Q, et al. Chronic stress suppresses the expression of cutaneous hypothalamic-pituitary-adrenocortical axis elements and melanogenesis. PLoS One 2014; 9 : e98283.

18. Boukamp P, Petrussevska RT, Breitkreutz D, et al. Normal keratinization in a spontaneously immortalized aneuploid human keratinocyte cell line. J Cell Biol 1988; 106: 761-71.

19. Slominski AT, Manna PR, Tuckey RC. Cutaneous glucocorticosteroidogenesis: securing local homeostasis and the skin integrity. Exp Dermatol 2014; 23: 369-74.

20. Slominski AT, Zmijewski MA. Glucocorticoids inhibit wound healing: novel mechanism of action. J Invest Dermatol 2017; 137: 1012-4.

21. Slominski A, Pisarchik A, Wortsman J, et al. Expression of hypothalamic-pituitary-thyroid axis related genes in the human skin. J Invest Dermatol 2002; 119: 1449-55.
22. Agretti P, De Marco G, De Servi M, et al. Evidence for protein and mRNA TSHr expression in fibroblasts from patients with thyroid-associated ophthalmopathy (TAO) after adipocytic differentiation. Eur J Endocrinol 2005; 152: 777-84. 